\title{
openheart Left ventricular longitudinal shortening: relation to stroke volume and ejection fraction in ageing, blood pressure, body size and gender in the HUNT3 study
}

To cite: Støylen A, Dalen H, Molmen HE. Left ventricular longitudinal shortening: relation to stroke volume and ejection fraction in ageing, blood pressure, body size and gender in the HUNT3 study. Open Heart 2020;7:e001243. doi:10.1136/ openhrt-2020-001243

Received 16 January 2020 Revised 4 June 2020 Accepted 7 August 2020
D) Check for updates

(c) Author(s) (or their employer(s)) 2020. Re-use permitted under CC BY-NC. No commercial re-use. See rights and permissions. Published by BMJ.

${ }^{1}$ Faculty of Medicine, Dept of Circulation and Medical Imaging, NTNU, Norwegian University of Science and Technology, Trondheim, Norway

${ }^{2}$ Cardiology, St. Olav University Hospital, Trondheim, Norway ${ }^{3} \mathrm{MI}$ Lab and Department of Circulation and Medical Imaging, NTNU, Trondheim, Norway

${ }^{4}$ Cardiology, Levanger Hospital, Levanger, Norway

${ }^{5}$ Asgardstrand General Practice, Horten, Norway

${ }^{6}$ Division of Medicine,

Department of Endocrinology, Morbid Obesity Centre, Vestfold Hospital Trust, Tonsberg, Norway

Correspondence to Professor Asbjørn Støylen; asbjorn.stoylen@ntnu.no

\section{ABSTRACT}

Background Aims of this cross-sectional study were to assess: the relative contribution of left ventricular (LV) systolic long-axis shortening (mean mitral annular plane systolic excursion, MAPSE) to stroke volume (SV), the mechanisms for preserved ejection fraction (EF) despite reduced MAPSE, the age dependency of myocardial volume and myocardial systolic compression.

Methods Linear dimensions and longitudinal and crosssectional M-modes were acquired in 1266 individuals without history of heart disease, diabetes or known hypertension from the third wave of the Nord-Trøndelag Health Study. Measurements were entered into a halfellipsoid LV model for volume calculations, and volumes were related to age, body size (body surface area, BSA), sex and blood pressure (BP).

Results Mean BP and proportion with hypertensive values increased with increasing age. MAPSE contributed to $75 \%$ of SV, with no relation to age or BSA as both MAPSE and SV decreased with increasing age. LV end-diastolic volume (LVEDV) and SV increased with BSA and decreased with higher age; EF was not related to age or BSA. Myocardial volume increased with higher age and BSA, with an additional gender dependency. The association of age with myocardial volume was not significant when corrected for $\mathrm{BP}$, while both systolic and diastolic BP were significant associated with myocardial volume. Myocardial compression was less than $3 \%$.

Conclusions MAPSE contributes approximately $75 \%$ and short axis shortening $25 \%$ to SV. Both decline with age, but their percentage contributions to SV are unchanged. EF is preserved by the simultaneous decrease in LVEDV and SV. Myocardial volume is positively associated with age, but this is only related to higher $\mathrm{BP}$, which may have implications for BP treatment in ageing. The myocardium is near incompressible.

\section{INTRODUCTION}

Systolic deformation of the left ventricular (LV) comprises two deformations, longitudinal and transversal (short-axis) decrease, ${ }^{12}$ as shown in figure 1 . The systolic reduction in total volume is a function of both. There have been various reports on the relative importance of the two components. ${ }^{3-10}$

\section{Key questions}

What is already known about this subject?

- The relative contributions of systolic short-axis and long-axis shortening to stroke volume is still disputed.

- Long-axis shortening decreases with increasing age, but ejection fraction is preserved, although the mechanism is unresolved.

- Left ventricular (LV) wall thickness increases with age, but as the ventricular length decrease simultaneously, the effect on LV mass is uncertain.

- The myocardium is considered relatively incompressible, but the degree of incompressibility is little studied.

What does this study add?

- This study indicates that long-axis shortening is the main contributor to the stroke volume. Even though long-axis shortening declines with age, end-diastolic volume and stroke volume decreases simultaneously preserving both the contribution of long-axis shortening to stroke volume, and the ejection fraction, while there is no increase in short-axis function.

- As there is increased wall thickness but decreased LV length, volume and mass do not increase with age per se, but the increase is relative to higher blood pressure by age.

- There is little systolic compression of the myocardium, and the compressibility is not related to age blood pressure or body size. This is important for the understanding of the geometry of myocardial systolic deformation.

With increasing age, there is a reduction in the LV long-axis shortening, both absolute shortening which equals mitral annular plane systolic excursion (mean mitral annular plane systolic excursion, MAPSE), and relative shortening; global longitudinal strain, except in one larger meta-analysis, ${ }^{11}$ (probably due to between-study variability), while the ejection fraction (EF) is preserved. ${ }^{12-18}$ It has been suggested that this is due to increased systolic short-axis contraction, ${ }^{19-22}$ but this 


\section{Key questions}

How might this impact on clinical practice?

- The study shows the importance of long-axis shortening as a measure of LV function and the age-dependent decline of myocardial function. Mean mitral annular plane systolic excursion is a useful measure of LV useful and contributes to $75 \%$ of stroke volume independent of age, gender and body size. It also elucidates that geometric changes renders ejection fraction less useful in age and hypertension related hypertrophy.

- Furthermore, it is little effect of age on myocardial volume, although the ventricles become more spherical with higher age, so LV mass measurements should not be based on short-axis measures alone. The increase in LV volume with higher age is an effect of higher blood pressure with age, not ageing per se.

- This may indicate that treatment of moderate hypertension is to be important also in higher age groups, especially in the presence of LV hypertrophy.

is inconsistent with the well-documented unchanged endocardial fractional shortening (FS) with increasing age. ${ }^{23-28}$ Also, studies have demonstrated that all three strain components may be reduced in hypertrophic conditions, ${ }^{29-32}$ so increased short-axis function is not a prerequisite for preservation of $\mathrm{EF}$ in the presence of reduced long-axis shortening. We have previously found lower longitudinal, circumferential and transmural (radial) strain with increasing age in the present study population. ${ }^{1}$

LV wall thickens with increasing age. ${ }^{16} \quad 23 \quad 26-28 \quad 33$ Contrarily to as previously believed, this does not necessarily mean an increase in LV mass, ${ }^{23} 2534$ because LV length decreases with age. ${ }^{28}{ }^{35}$ Furthermore, most study populations show an increase of blood pressure (BP) with age, which must be taken into account.

The myocardium is generally considered fairly incompressible. ${ }^{36}$ We have previously shown that by strain measurements that systolic compression was very low. ${ }^{1}$ The compressibility is pertinent for the relation between outer volume decrease and stroke volume (SV) (figure 1).

The objectives of this study were to assess:

1. The relative contribution of long-axis and short-axis shortening to SV.

2. The relations of LV end-diastolic volume (LVEDV), SV and $\mathrm{EF}$ with age, BP, sex and body size, and the mecha-

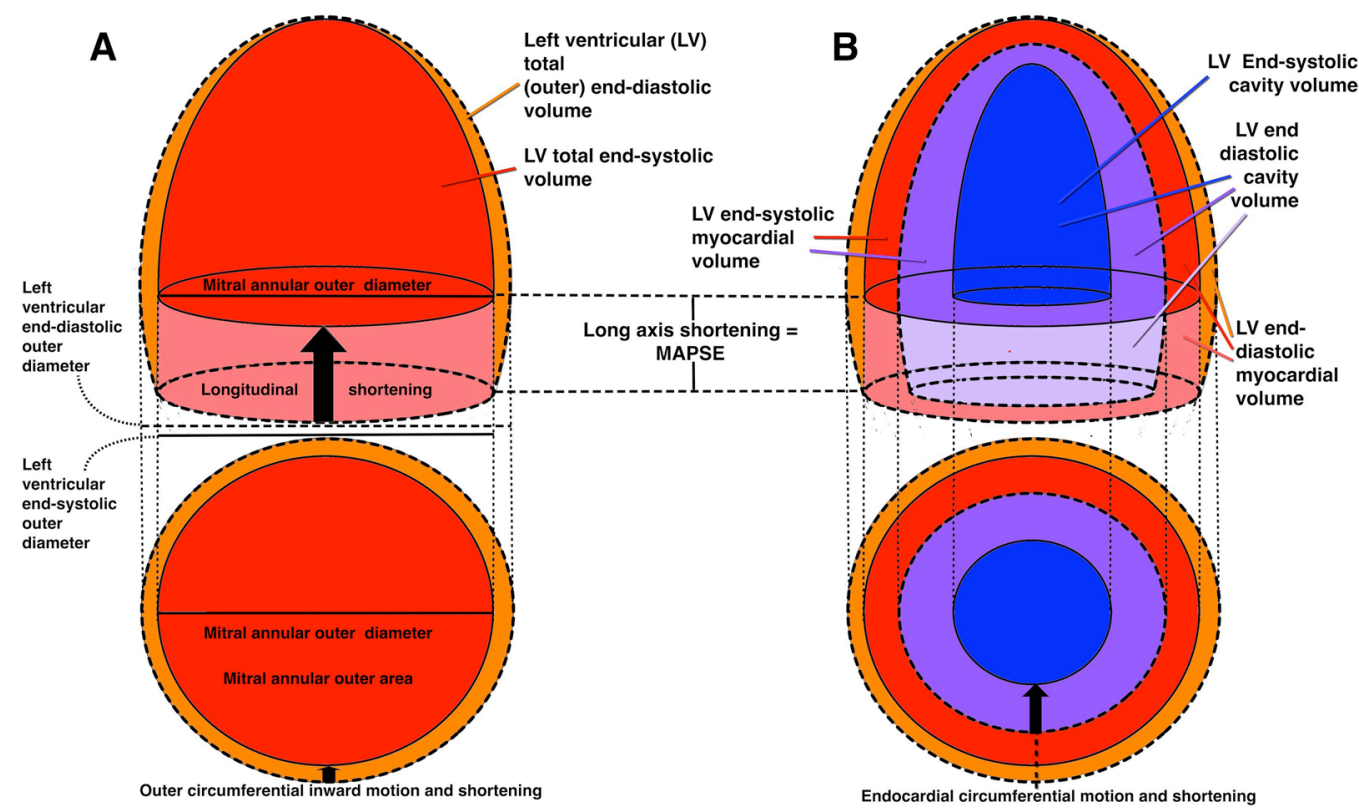

Figure 1 Schematic diagram of the systolic deformation of the left ventricle. Systolic contours are shown by the broken lines, diastolic by the unbroken. (A) Outer, or total volumes. total volume=myocardial vol+cavity vol. The ventricle reduces both length and outer diameter, and the total volume decreases as a function of both deformations (orange and light red). if the myocardium is incompressible, the myocardial volume is the same in both diastole and systole. thus, diastolic cavity volume=total diastolic volume - myocardial volume and systolic cavity volume=total systolic volume - myocardial volume. Stroke volume=diastolic cavity volume - systolic cavity volume, which then must be diastolic total volume - systolic total volume. The systolic decrease of the outer volume must then equal the stroke volume (SV), irrespective of the internal configuration changes of the cavity and myocardium, which are irrelevant. The longitudinal shortening (MAPSE) $X$ the cross-sectional mitral annular area makes up the fraction of the SV due to long axis shortening (light red cylinder). The remaining systolic outer volume reduction must be due to outer diameter shortening (orange). (B)Cavity and myocardial volumes. both total and cavity volumes can be measured by diameter and length in systole and diastole as detailed in the online supplemental appendix. Myocardial volume is total volume - cavity volume in end-diastole and end-systole, respectively. myocardial systolic compression is myocardial diastolic - systolic volume. Stroke volume = end-diastolic - end systolic cavity volume. The cavity decreases due to decrease of both diameter and length (dark and light violet), but the inner diameter decrease (endocardial fractional shortening) is due to both outer diameter shortening, and myocardial thickening, the latter being mainly a function of myocardial longitudinal shortening. MAPSE, mean mitral annular plane systolic excursion. 
nism for preserved $\mathrm{EF}$ despite reduced long-axis shortening with increasing age.

3. Changes in myocardial volume with age.

4. The amount of systolic myocardial systolic compression (MC) .

\section{METHODS}

All basic measurements in this study have been published previously, ${ }^{1}{ }^{18} 28$ and are from the echocardiographic substudy of the third wave of the Nord-Trøndelag Health Study (HUNT3) echo substudy. The basic linear measures are given in the online supplemental appendix. In the present study, measures are used in a half ellipsoid model to calculate volumes, as comprehensively described in the online supplemental appendix.

\section{Study subjects}

The study population was recruited from the HUNT3 Study and has been extensively described in previous papers. ${ }^{142837}$ Briefly, of 50839 participants of the HUNT3 Study a random sample restricted to two communities was invited to the echocardiographic study. After exclusion of subjects with a history of heart disease, hypertension or diabetes 1296 were included. After echocardiography, another 30 individuals with significant pathology on the echocardiogram were excluded. Thus, the study consisted of 1266 subjects with age 19-89 years. BP was measured three times by trained staff members using a Dinamap 845XT (GE Healthcare, Milwaukee, Wisconsin, USA). Measurements were made after 2 min of rest with the arm on a table, and the average of the second and third measurements was used in the analyses. Basic characteristics are given in table 1 .

All subjects gave their written informed consent to participate in the study.

\section{Echocardiography}

Subjects were examined in the left lateral supine position with a Vivid 7 scanner (version BT06, GE Ultrasound,
Horten, Norway), with phased-array matrix transducers (M3S and M4S). One experienced echocardiographer (HD) did all the examinations, which included parasternal long-axis and apical two-chamber and fourchamber views. For each view, at least three consecutive cardiac cycles were recorded during quiet respiration. Mean B-mode frame rate was 44 frames per second.

Diastolic wall length (WL) was measured as the straight lines from epicardial apex to the mitral points in the four walls in diastole (figure 2A), and mean WL was calculated from four walls. This was converted to outer and inner LV diastolic length (LVLd and LVILd, respectively) as described in the online supplemental appendix. MAPSE was measured from reconstructed longitudinal M-mode of the mitral annulus in the same four points, and likewise averaged. Septal and posterior wall thicknesses in diastole (IVSd and LVPWd respectively) and systole (IVSs and LVPWs, respectively) and diastolic and systolic LV internal chamber diameters (LVIDd and LVIDs) were all measured in parasternal M-mode at the tip of the mitral leaflets (close to the papillary muscles) in end diastole (figure 2B). Values were averaged to diastolic and systolic wall thicknesses (WTd and WTs, respectively), and outer LV diastolic and systolic diameters (LVEDd and LVEDs) were calculated from LVIDd, LVIDs WTd and WTs. Endocardial FS was calculated as FS $=($ LVIDd - LVIDs $)$ / LVIDd, and external FS from external diameters the same way. Derivation of remaining linear measures for volume calculations is described in the online supplemental appendix.

\section{Calculations and statistics}

Both calculations and statistics were done in SPSS V.23 (IBM). Ventricular volumes were calculated from the basic and derived linear dimensions in a half-ellipsoid model as described in detail in the online supplemental appendix. Briefly, a symmetrical half ellipsoid was assumed, with homogeneous wall thickness around the circumference, but with a wall thickness in the apex

\begin{tabular}{|c|c|c|c|c|c|c|c|}
\hline Age & $\mathbf{N}$ & Height (m) & Weight (Kg) & BSA $\left(m^{2}\right)$ & BMI & $\mathrm{SBP}(\mathrm{mm} \mathrm{Hg})$ & DBP $(\mathrm{mm} \mathrm{Hg})$ \\
\hline \multicolumn{8}{|c|}{ Women } \\
\hline$<40$ & 208 & $1.67(0.11)$ & 72.5 (17.4) & $1.80(0.17)$ & $25.5(4.6)$ & $119(12)$ & $68(9)$ \\
\hline $40-60$ & 336 & $1.65(0.12)$ & 71.7 (12.5) & $1.80(0.15)$ & $26.0(4.0)$ & $126(16)$ & $72(11)$ \\
\hline$>60$ & 119 & $1.62(0.12)$ & $70.0(10.8)$ & $1.75(0.13)$ & $26.3(3.9)$ & $143(20)$ & $75(10)$ \\
\hline Total & 663 & $1.65(0.11)$ & $71.6(14.0)$ & $1.79(0.15)$ & $25.9(4.2)$ & $127(17)$ & $71(19)$ \\
\hline \multicolumn{8}{|c|}{ Men } \\
\hline$<40$ & 126 & $1.81(0.06)$ & 86.1 (13.8) & $2.06(0.16)$ & $26.5(4.1)$ & $128(11)$ & $71(10)$ \\
\hline $40-60$ & 327 & $1.80(0.08)$ & 89.6 (39.5) & $2.07(0.16)$ & $27.2(3.4)$ & 132 (12) & $78(10)$ \\
\hline$>60$ & 150 & $1.76(0.06)$ & $82.4(14.1)$ & $1.98(0.14)$ & $26.4(2.9)$ & $140(17)$ & $78(10)$ \\
\hline Total & 603 & $1.79(0.07)$ & $87.1(30.7)$ & $2.05(0.16)$ & $26.8(3.5)$ & $133(14)$ & $77(10)$ \\
\hline All & 1266 & $1.72(0.11)$ & $79.0(24.7)$ & $1.91(0.20)$ & $26.3(3.9)$ & $130(16)$ & $74(11)$ \\
\hline
\end{tabular}

Measurements are mean (SD).

BMI, body mass index; BSA, body surface area; DBP, diastolic blood pressure; SBP, systolic diastolic blood pressure. 


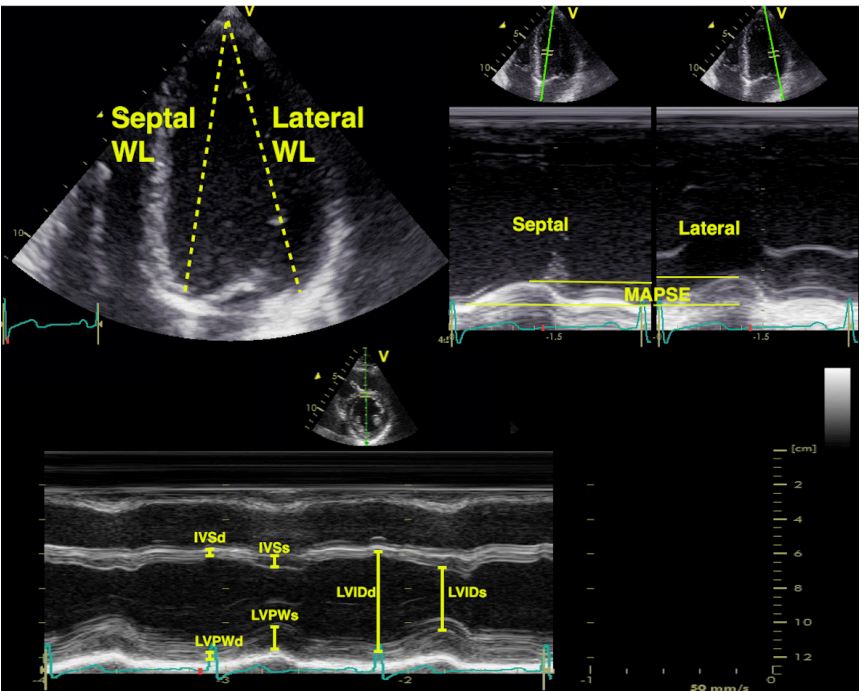

Figure 2 Measurements for the volume calculations. Top left: wall length (WL) measurements taken as a straight line from the epicardial apex to the mitral annulus, shown for the septal and lateral WL measurements in the four-chamber view. Top right, the reconstructed M-modes from the same mitral annular points, showing the apical motion (MAPSE) equal to the left ventricular shortening. In the study, the mean of four walls from four-chamber to two-chamber views were used. MAPSE: mean mitral annular plane systolic excursion; IVSd: diastolic septum thickness; IVSs: systolic septum thickness; LVPWd: diastolic posterior wall; LVPWs: systolic posterior wall; LVIDd: internal diastolic (cavity) diameter; LVIDs: internal systolic (cavity) diameter.

of $50 \%$ of the midwall thickness, in both diastole and systole. The calculations resulted in two ellipsoids, a total (outer) volume in diastole and systole, and an inner ellipsoid, representing the cavity. LV end-diastolic and endsystolic cavity volumes, respectively (LVEDV and LVESV) were the volumes of the internal ellipsoid. Myocardial diastolic and systolic volumes (MVd and MVs) were the differences between total volume and cavity volumes in systole and diastole, respectively, and MC was the relative difference between MVd and MVs. SV and EF was calculated from cavity volumes. Finally, the contribution of MAPSE to SV (MAPSE_SV) was calculated as MAPSE $\mathrm{x}$ mitral annular outer cross-sectional area, as shown in figure 1 . The remaining part of the SV would be contribution by the short-axis function.

Means and SD are given as data were (near) normally distributed. The significance of differences between measures were tested by one-sample student's t-test, between genders by independent samples t-test, differences between age groups ( $<40$ years, $40-60$ and $>60$ years) were tested by one-way analysis of variance, with Bonferroni post hoc comparisons. Correlations were tested by full and partial (within BP groups) Pearson's correlation and multiple linear regression.

Reproducibility of the basic measures have been published previously. ${ }^{28} 38$ Shortly, the inter analyser testretest mean error was $10 \%$ for wall thickness and $5 \%$ for LV diameter. Coefficients of repetition (CoR) were 1.8
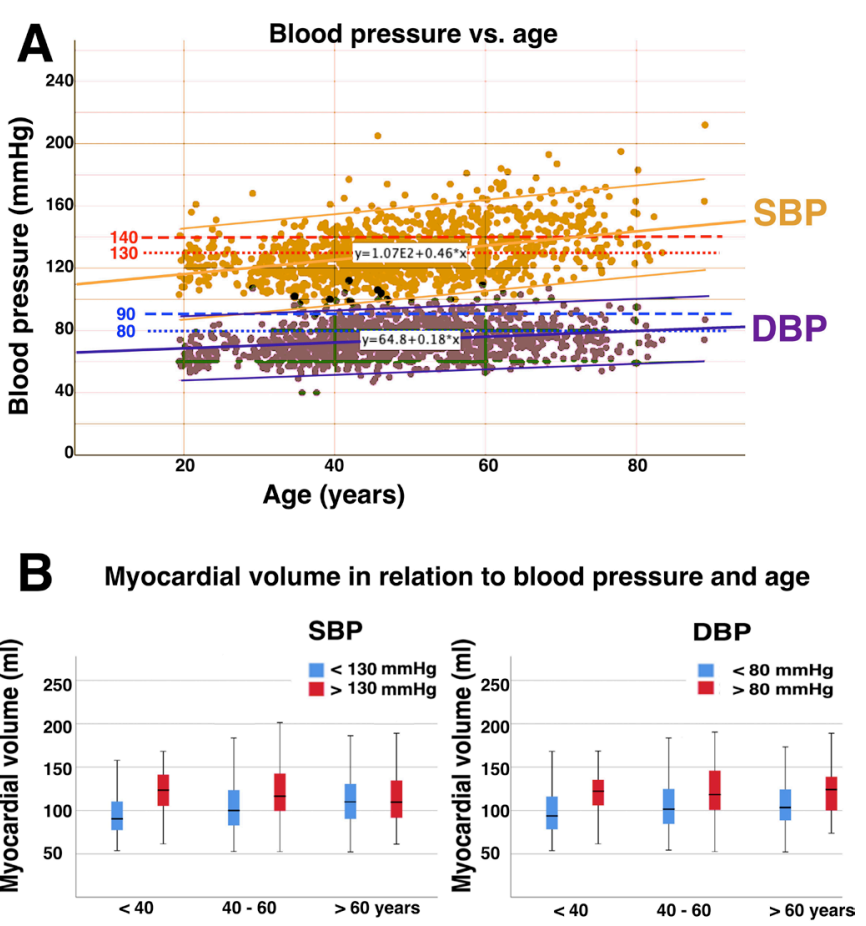

Figure 3 (A) Blood pressure in the study population. Upper limits of high normal blood pressure $(130 / 80 \mathrm{~mm} \mathrm{Hg})$ and lower limit for hypertension $(140 / 90 \mathrm{~mm} \mathrm{Hg}$ ) are indicated by the dotted lines. The study shows both increasing mean BP with increasing age, as well as increasing proportions with hypertensive values. (B) Myocardial volume in relation to SBP and DBP and age. Here, cut-off values of 130/80 $\mathrm{mm} \mathrm{Hg}$ (high normal) was used, but as seen in the text, the volumes differed only 2-4 $\mathrm{mL}$ for a cut-off of $140 / 90 \mathrm{~mm} \mathrm{Hg}$. There was no significant correlation of myocardial volume with age within BP groups. BP, blood pressure; DBP, diastolic blood pressure; SBP, systolic blood pressure.

$\mathrm{mm}$ for IVSd, $2.4 \mathrm{~mm}$ for LVPWd and $5.4 \mathrm{~mm}$ for LVIDd. Mean error was $3 \%$, and CoR was $1.6 \mathrm{~mm}$ for global MAPSE, and $3 \%$, and $0.7 \mathrm{~cm}$ for mean WL, respectively.

\section{RESULTS}

\section{Study subjects}

Age did not correlate with body mass index (BMI) and were only borderline significant associated with BSA $(\mathrm{R}=0.06, \mathrm{p}=0.04)$. When multiple correlations were taken into account the association was non-significant. BSA was $15 \%$ higher in men than women, while BMI was only $3 \%$ higher in men (both $\mathrm{p}<0.001$ ).

None of the study population had a history of known hypertension (or antihypertensive treatment), diabetes or heart disease, and those with indications of heart disease on echo were excluded. However, it is evident from table 1 that there was some untreated hypertension in the material, most common in the highest age group. Both systolic (SBP) and diastolic BP (DBP) correlated with age, $\mathrm{R}=0.40$ and 0.24 , respectively (both $\mathrm{p}<0.001$ ). SBP was significantly different between all three age groups, while DBP was not significant between the two oldest age groups in post hoc analysis. In the three age 
Table 2 Conventional volumes and functional measures, and the contribution of long axis shortening to stroke volume according to age and gender

\begin{tabular}{|c|c|c|c|c|c|c|c|c|}
\hline Age & MAPSE (cm) & MAPSE_SV (mL) & MAPSE_SV (\%) & LVEDV (mL) & LVESV (mL) & SV (mL) & EF (\%) & FS (\%) \\
\hline \multicolumn{9}{|c|}{ Women } \\
\hline$<40$ & $1.73(0.20)$ & $56.5(9.9)$ & $75.4(11.9)$ & $111.6(21.6)$ & $87.0(19.1)$ & $76.3(16.7)$ & $68(6)$ & $36.6(6.1)$ \\
\hline $40-60$ & $1.58(0.23)$ & $53.3(11.7)$ & $74.9(13.5)$ & $106.9(21.7)$ & $92.8(19.6)$ & $72.7(17.0)$ & $68(7)$ & $36.5(6.9)$ \\
\hline$>60$ & $1.33(0.22)$ & $45.2(10.1)$ & $72.0(21.9)$ & $97.9(19.7)$ & $95.6(18.9)$ & $65.4(16.9)$ & $66(9)$ & $36.0(9.1)$ \\
\hline Total & $1.58(0.26)$ & $52.9(11.5)$ & $74.6(14.9)$ & $106.8(21.8)$ & $91.4(19.6)$ & $72.6(17.3)$ & $68(8)$ & $36.4(7.1)$ \\
\hline \multicolumn{9}{|c|}{ Men } \\
\hline$<40$ & $1.72(0.22)$ & $70.1(74.9)$ & $74.9(14.2)$ & $144.8(30.5)$ & $87.0(19.1)$ & $96.1(22.9)$ & $66(8)$ & $35.5(6.9)$ \\
\hline $40-60$ & $1.58(0.22)$ & $65.1(14.2)$ & $72.8(14.8)$ & $138.1(31.1)$ & $92.8(19.6)$ & $92.2(23.8)$ & $67(8)$ & $35.8(7.4)$ \\
\hline$>60$ & $1.45(0.21)$ & $60.3(15.6)$ & $74.9(19.0)$ & $126.3(33.7)$ & $95.6(18.9)$ & $84.1(25.7)$ & $66(9)$ & $36.0(8.0)$ \\
\hline Total & $1.58(0.24)$ & $64.9(14.7)$ & $73.8(15.8)$ & $136.6(32.2)$ & $91.4(19.6)$ & $91.0(24.4)$ & $67(8)$ & $35.8(7.5)$ \\
\hline All & $1.58(0.25)$ & $61.5(13.0)$ & $75.2(12.8)$ & $121.1(31.1)$ & $87.0(19.1)$ & $81.4(22.9)$ & $67(8)$ & $36.1(7.3)$ \\
\hline
\end{tabular}

EF, ejection fraction; FS, fractional endocardial diameter shortening; LVEDV, left ventricular end-diastolic (cavity) volume; LVESV, left ventricular end-systolic (cavity) volume; MAPSE, mean mitral annular plane systolic excursion; SV, stroke volume; MAPSE SV, The contribution to SV of MAPSE.

groups, $<40,40-60$ and $>60$ years, respectively, $7 \%, 18 \%$ and $44 \%$ had SBP $>140 \mathrm{~mm} \mathrm{Hg}$, and $24 \%, 40 \%$ and $65 \%$ SBP $>130 \mathrm{~mm} \mathrm{Hg}, 3 \%, 9 \%$ and $10 \%$ had DBP $>90 \mathrm{~mm}$ $\mathrm{Hg}, 12 \%, 31 \%$ and $59 \%$ had DBP $>80 \mathrm{~mm} \mathrm{Hg}$. The BPs are shown in figure 3 . The proportions with elevated BP at screening were slightly higher in men.

\section{MAPSE contribution to SV}

MAPSE and MAPSE_SV are given in table 2. MAPSE_SV was on the average $61.5 \mathrm{~mL}, 75 \%$ of the total SV. This means that external FS contributed $25 \%$ to the total SV. As shown previously, ${ }^{18}$ MAPSE correlated negatively with age $(\mathrm{R}=-0.50)$, weakly positively with BSA $(\mathrm{R}=0.12, \mathrm{p}<0.001)$, and there was no significant sex difference. Furthermore, MAPSE correlated negatively with $\mathrm{BP}(\mathrm{R}=-0.27$ and -0.24 for SBP and DBP, respectively (both $\mathrm{p}<0.001$ ). In linear regression analysis only age $(\beta=-0.47, \mathrm{p}<0.001)$ and DBP $(\beta=-0.13, p<0.001)$ but not SBP, were significantly associated with MAPSE.
MAPSE_SV in \% of SV did not correlate with age, BSA, SBP nor DBP.

\section{LV cavity volumes, SV and EF}

All measured and derived linear measurements used for calculations are given by age and gender in online supplemental table 1 and in the online supplemental appendix, most have been described previously. ${ }^{28}$ Conventional functional measures and model derived cavity volumes are given in table 2 . The linear measures, both basic and derived, were all near normally distributed.$^{28}$ Volumes, however, were moderately skewed as shown in figure 4, with skewnesses of $0.71,0.65$ and 0.55 for LVEDV, SV and MVd, respectively.

BSA correlated strongly with LVEDV, LVESV and SV with $R$ of $0.58,0.48$ and 0.50 , respectively (all $p<0.001$ ), but not with EF and FS. There were significant sex differences $(p<0.001)$ for all volumes, both sex and BSA remained significant in linear regression. The sex difference in EF
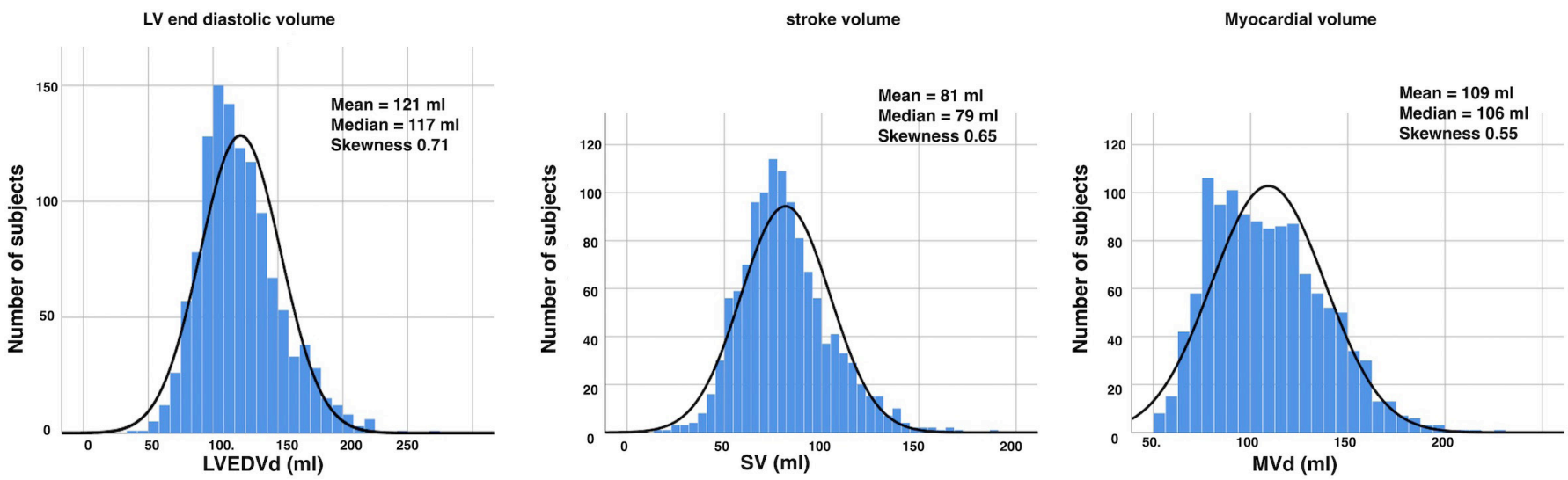

Figure 4 Distribution of the model derived volumes. The distribution is skewed, while the basic measures were not, so the skewness may be due to the model. LV, left ventricular; LVEDV; left ventricular end-diastolic (cavity) volume; MVd; end-diastolic myocardial volume; SV; stroke volume. 


\begin{tabular}{|c|c|c|c|c|c|}
\hline Age (years) & MVd (mL) & MVs (mL) & $M C(m L)$ & MC (\% of MVd) & MC (\% of SV) \\
\hline \multicolumn{6}{|c|}{ Women } \\
\hline$<40$ & $87.0(19)$ & $83.7(17.8)$ & $3.3(9.9)$ & $3.1(11.1)$ & $5.2(14.6)$ \\
\hline $40-60$ & $92.8(19.6)$ & $89.3(18.7)$ & $3.5(11.2)$ & $3.0(11.9)$ & $5.0(16.2)$ \\
\hline$>60$ & $95.6(18.9)$ & $93.3(18.0)$ & $2.2(10.3)$ & $1.6(11.1)$ & $4.0(16.1)$ \\
\hline Total & $91.4(19.6)$ & $88.2(18.6)$ & $3.2(10.7)$ & $2.8(11.5)$ & $4.9(15.7)$ \\
\hline \multicolumn{6}{|c|}{ Men } \\
\hline$<40$ & $125.3(23.6)$ & $122.3(23.3)$ & $2.8(13.9)$ & $1.8(10.6)$ & $4.0(14.9)$ \\
\hline $40-60$ & $129.7(25.3)$ & $126.7(24.9)$ & $3.1(14.9)$ & $1.8(11.3)$ & $4.6(17.7)$ \\
\hline$>60$ & $128.2(26.8)$ & $123.8(26.8)$ & $4.4(13.0)$ & $3.1(10.0)$ & $5.3(17.9)$ \\
\hline Total & $128.4(25.3)$ & $125.1(25.1)$ & $3.4(14.2)$ & $2.1(10.9)$ & $4.6(17.2)$ \\
\hline All & $101.4(27.9)$ & $98.2(27.4)$ & $3.1(11.6)$ & $2.6(11.0)$ & 4.8 (16.4) \\
\hline
\end{tabular}

MC, myocardial systolic compression; MVd, left ventricular myocardial diastolic volume; MVs, myocardial systolic volume; SV, stroke volume.

of $1 \%$ point was also significant $(\mathrm{p}<0.005)$, despite being of low clinical interest, while FS was not related to gender, as also shown previously. ${ }^{28}$

Age was weakly, negatively correlated with LVEDV and SV with $\mathrm{R}-0.12$ and -0.13 , respectively (both $\mathrm{p}<0.001$ ), but showed no association with LVESV and EF. In linear regression with BP, age was negatively associated with LVEDV in multiple linear regression $(\beta=-0.18, p<0.001)$, but only DBP was borderline, weakly independently associated with LVEDV. (univariate $\mathrm{R}=0.09, \mathrm{~B}=0.09, \mathrm{p}=0.02$ ). FS, LVESV, SV and EF did not correlate significantly with SBP or DBP, while external FS was $12.8 \%$, and correlated negatively with age $(\mathrm{R}=-0.15)$ but not with $\mathrm{BP}$.

\section{Myocardial volumes and compressibility}

Myocardial volumes and systolic compression are given in table 3. LV MVd was on the average $101 \mathrm{~mL}$, with a significant sex difference $(\mathrm{p}<0.001)$. MVd correlated strongly with BSA $(\mathrm{R}=0.70, \mathrm{p}<0.001)$, but both BSA and sex were independently associated with MVd in multiple linear regression ( 10.50 and 0.32 , respectively, both $\mathrm{p}<0.001$ ).

MVd was also significantly different between age groups $(p<0.001)$. However, in post hoc analysis, the difference between the two oldest age groups was not significant, and the correlation with age was weak $(\mathrm{R}=0.14, \mathrm{p}<0.001)$. Furthermore, there were no significant partial correlations with age controlling for $\mathrm{BP}$ groups, neither with cut-off 140/90 nor 130/80 mm Hg. Correlations with SBP and DBP was stronger with R 0.39 and 0.24 , respectively (both $\mathrm{p}<0.001$ ). In multiple linear regression, both SBP and DBP remained significant associated with MVd ( $\beta=0.15$ and 0.20 , respectively, both $p<0.001)$, while age did not. For SBP, MVd was $118 \mathrm{~mL}$ in men vs $102 \mathrm{~mL}$ in women for a cut-off of $130 \mathrm{~mm} \mathrm{Hg}$, and correspondingly 120 vs 106 for a cut-off of $140 \mathrm{~mm} \mathrm{Hg}(\mathrm{p}<0.001)$. For DBP, MVD was 122 vs 104 in men and women for a cut-off of 80 $\mathrm{mm} \mathrm{Hg}$, and 124 vs 108 for $90 \mathrm{~mm} \mathrm{Hg}$ correspondingly $(p<0.001)$. The distribution of MVd vs age groups and BP is shown in figure $3 \mathrm{~B}$.
MC was low, mean $3.1 \mathrm{~mL}$, or $2.6 \%$ of MVD, $4.8 \%$ of SV. There were no correlations with age, BSA, SBP or DBP.

\section{DISCUSSION}

The main findings of the study were:

1. Long-axis shortening contributed to $75 \%$ of the total $\mathrm{SV}$, according to this model, and the proportion was independent of BSA, BP and age.

2. LVEDV and SV both decreased with higher age, and the ventricle became more spherical. EF remained unchanged, and so did endocardial FS. Both gender and BSA were associated with LV volumes, SV and myocardial volume.

3. Myocardial volume increased by age, but both SBP and DBP increased with higher age, and were associated with increasing myocardial volume. The age dependency of myocardial volume was not significant when adjusted for the higher BP with higher age.

4. MC was small, mean $2.6 \%$ of end-diastolic myocardial volume, $4.8 \%$ of SV. This compression was independent of BSA, gender, age and BP.

\section{Study subjects}

The average BMI was $25 \mathrm{~kg} / \mathrm{m}^{2}$, indicating that half the population was overweight. However, this is a common finding in western countries today, and compared with the general HUNT3 population the values for BMI were slightly lower in the echocardiographical substudy. ${ }^{39} \mathrm{BMI}$ was similar in the study by Hees $e t a l^{35}$ and only slightly lower in the NORRE study. ${ }^{16}$ Despite exclusion of subjects with known hypertension, there was a proportion of hypertensives in this population. Even though the measured BPs being spot pressures at baseline examinations, the results, especially in view of the relation with myocardial volume, indicate a higher percentage of hypertensives with age.

Due to the large size, measurements are done with a limited set of simplified measurements. As the 
conventional LV dimensions and FS in this material (data in the online supplemental appendix) are in line with others, ${ }^{23} 26274041$ the population seems to be fairly representative. The results are based on an ellipsoid model, with limited validity, as discussed below. Basically, we assume the ventricle to remain ellipsoid with age. This is, granted, an additional assumption. The model itself assumes a symmetry that is not accurate, and the skewness of the true LV shape may well change with age. However, the actual shape of the ellipsoid changed, the external diameter increasing, the length decreasing. This is evident from the basic measurements themselves, and not only a function of the model.

\section{MAPSE contribution to SV}

The concept that the heart works mainly by the AV-plane motion, is not new, being proposed already by Leonardo da Vinci, ${ }^{42}$ and supported by experimental work in the $1930 \mathrm{~s},{ }^{43}$ experimental CT studies ${ }^{44}$ from the 1980 s, observational imaging studies in humans ${ }^{3}$ and lastly by cardiac magnetic resonance (CMR) imaging. ${ }^{5}$ Some has suggested that all of the SV is due to AV-plane motion, ${ }^{3}$ as the myocardium is incompressible, and that the decrease of LV diameter is due to wall thickening alone. However, with an external FS of $12.8 \%^{1}$ consistent with other findings, ${ }^{2}$ there has to be a component due to cross-sectional shortening as well. ${ }^{45}$ The contribution of MAPSE has been estimated to be as high as $83 \%$ in an echo study, ${ }^{4}$ and around $60 \%$ in recent CMR studies., ${ }^{86}$ with the present results in-between these findings.

The total (outer) volume consists of the myocardium and the cavity. An incompressible myocardium will only change shape, not volume in systole. Thus, the outer volume systolic decrease must equal the decrease of the cavity volume, which is the SV. In the present study, the compressibility of the myocardium was low, so this approximation seems fair. But this means that the outer diameter shortening, and the longitudinal shortening are the determinants of the SV, as illustrated in figure 1. Thus, the internal relations of diameters, circumferences, lengths and cavity volumes are, in fact, irrelevant. $\mathrm{SV}$, calculated from cavity volumes, will still give the absolute SV, but from the argument above, must equal outer volume decrease.

The contribution of MAPSE to SV equals the crosssectional area of the base (external mitral annular area) $x$ MAPSE, equal to the volume of a cylinder as shown in figure 1. In calculating the area, we used the systolic $\mathrm{LV}$ diameter rather than the diastolic, as this is probably closer to the annular diameter; LV external diameter is compressible while the mitral annulus is probably not. However, we included the whole annular thickness, as the SV relates to outer volume decrease. Using only the annular orifice diameter, as some authors have done ${ }^{7}$ will be erroneous, and result in too low contribution of MAPSE to SV.

Shortening of the outer circumference and diameter during systole must be due to circumferential fibre shortening, while both midwall and endocardial circumferences move inwards partly as a function of wall thickening, which again is partly a function of longitudinal shortening. ${ }^{134847}$ This inward motion results in shortening of the midwall and endocardial circumferences and diameters and would have been present even without circumferential shortening. Thus, only outer circumferential shortening is independent of longitudinal shortening ${ }^{1}$ figure 1C. Mean speckle tracking derived circumferential strain ${ }^{10}$ is equivalent to midwall circumferential strain, and thus longitudinal shortening dependent.

The relative contribution of MAPSE to SV remained constant with higher age, due to the simultaneously lower MAPSE and SV. As the absolute value of MAPSE decreases with increasing age, the relation between MAPSE and EF changes with age, as already described by Emilsson $e t$ al. $^{12}$ MAPSE correlated with BP, but only DBP was independently associated with MAPSE, so this was not an afterload effect, but rather hypertensive myocardial changes. The SBP range was possibly too small to give a significant afterload effect.

MAPSE was only weakly correlated with BSA. Both LVLd and LVEDd increase in proportion to BSA, and the ratio between them is BSA independent, ${ }^{28}$ thus, MAPSE SV increases mainly by increased cross-sectional mitral annular area, as illustrated in figure 1 . The increase in MAPSE by higher BSA $^{18}$ will then be far less, explaining the relatively low BSA dependency of MAPSE.

\section{LV volumes and EF}

In this model of the LV, both diameter and length are taken into consideration, but the half ellipsoid model rests on assumptions. The most important assumption relates to geometric symmetry, which is not correct for the LV, but which is similar to the model by Stokke et al. ${ }^{10}$ As all the linear measures are normally distributed, while the model-based volumes are skewed, this may indicate a systematic error in the volumes. LVEDV and EF are higher than in the study by Kou et al, ${ }^{16}$ but means are still within the normal range for BSA indexed LVEDV given by Lang et al. ${ }^{33}$ Compared with the indexed LVIDd by $\mathrm{CMR},{ }^{35}$ values are fairly similar, while $\mathrm{LV}$ internal length is longer. MVd is slightly lower than the values for LV mass in the NORRE study, ${ }^{16}$ and even further below the CMR values by Hees. ${ }^{35}$ Those studies, however, do not indicate whether the values are normally distributed, but it may seem that our model may overestimate cavity volume and underestimate myocardial volume. Measurements in the present study are not intended to be normative, but systematic errors may be expected to be similar across ranges of BSA, genders, age groups and BPs, and the main issue of this study is the relations of measurements, not the values themselves.

In line with the expectations, LVEDV and SV increased with higher BSA, while EF, being SV/LVEDV was independent of BSA. Thus, also the proportional decrease of EDV and SV with higher age, can explain the unchanged 
EF by age, without any increase in cross-sectional function. Increased short-axis function is not a prerequisite for preserved $\mathrm{EF}$ in the presence of reduced long-axis shortening. LVIDd was independent of age, while both WT and LVEDd increased with age and LVL and LVILd decreased. Furthermore, there is little effect of age on myocardial volume, although the ventricles become more spherical with higher age, but t LVEDV and LVESV, being functions of internal length and diameter decreased, as described previously by CMR. ${ }^{35}$ As external FS decrease with increasing age, there is in fact no increase, but rather a decrease in cross-sectional LV function by age.

\section{Myocardial volume and relation to age and $\mathrm{BP}$}

Related to the aim of assessing systolic compressibility, we measured myocardial volumes, not mass. Systolic compression means that the volume decreases, while the mass is unchanged. Every imaging modality in reality measures myocardial volume and converts to mass by a constant (usually 1.05) based on data from validation studies against autopsies. Thus, all relations of MVd by age, BSA and BP, are the same as for LV mass.

LV mass has been assumed to increase with age, from the observed age-related wall thickening. However, in the NORRE study ${ }^{16}$ using two-dimensional (2D) echo, only a weak relationship between age and LV mass was found, and only in women. The relationship of age and BP in the material is not given. In the CMR study of Hees, ${ }^{35}$ there was a significant increase in BP with age. LV mass was found to be unchanged with age in women, as the effect of increased WT were neutralised by decreased LVL, while LV mass decreased with age in men. Apart from body size, BP was found to be the strongest predictor for LV mass. In our study, there was, despite the finding of reduced LVL, a modest, but significant increase in MVd in both sexes, but this seems to be due exclusively due to the effect of higher BP with higher age. This may indicate that the relatively modest age-related increase in BP is clinically significant. As the presence of LV hypertrophy is a prognostic factor in hypertensive patients, ${ }^{48}{ }^{49}$ treatment of moderate hypertension may be important also in the elderly. The diagnosis of LVH, however, should not be based on wall thickness alone.

\section{Myocardial systolic compression}

Strain interrelations will give an indication of the amount of incompressibility. ${ }^{1}$ However, different methods for calculating strains actually have different basic assumptions and technical solutions, so there is no 'gold standard' for strain, and thus, the relations between strain cannot be used for calculating the absolute compressibility. The volume calculation in the present paper shows about $2.6 \%$ compression of the myocardial volume, but this is still significant. Some degree of compression of capillaries and crypts is to be expected, but from this study, it is small. Our findings indicate that myocardial compression detracts only about 5\% from the SV. We have no data to support that compressibility in the transverse and longitudinal direction differ. If this is the case, the contribution of MAPSE to SV would remain the same even if compression had been taken into account.

\section{Study strength and limitations}

The main strength of the study is the size, being one of the largest normal echocardiography studies. The acquisitions and analyses are done by one single experienced echocardiographer, ensuring homogeneity of data.

M-mode measurements are prone to systematic errors. However, these systematic errors will be similar across age groups, body sizes and genders, so the differences may still be representative, even if values are not. Also, the population shows very little skewness in the basic measurements. ${ }^{182850}$ The main limitation is the volume model, as discussed previously, with a set of assumptions that are not completely realistic. This may cause the skewness of the volume data, but any systematic error may likewise be supposed to be fairly constant across age, BP and sex.

MAPSE is measured by reconstructed M-mode. This means the temporal resolution is the same as in 2D B-mode, which is low compared with real-time M-mode. As the movement of the mitral ring is near zero at end systole and end diastole, this is of little importance. Also, speckle tracking is done in B-mode with the same sampling rate, so reconstructed M-mode and speckle tracking are equivalent with respect to time resolution. In the present study, we have used the MAPSE from the mean of four walls, not six. This is because that was the original approach, ${ }^{4151}$ and we have previously shown that the difference in mean MAPSE from four and six walls was $0.7 \mathrm{~mm}$, while variability was larger with six walls. ${ }^{18}$

The effect of age is not true ageing, as this is a crosssectional, not a cohort study. The population in the HUNT Study is ethnically homogeneous. As the values are not intended to be normative, this is of less importance, but may still be important for gender, age and BP effects in ethnically different populations.

\section{CONCLUSIONS}

Long-axis shortening contributes to approximately $75 \%$ to SV, external diameter shortening to $25 \%$, and these proportions are independent of body size and age, as there are simultaneous changes in long-axis shortening and SV with changes in age and BSA. Both LVEDV and SV decreased with age, thus preserving EF with higher age, without any increase in short-axis function. In fact, the short-axis function also decreased with age. Myocardial volume increased with higher BP and did not increase with highere age after adjustment for BP, as wall thickness increased, but LV length decreased with increasing age. Finding of increasing LV volume with increasing BP may have implications for treatment of moderate hypertension, even in the elderly. The LV myocardium is near 
incompressible and the compressibility is independent of age, BSA or BP.

\section{Twitter Asbjørn Støylen @strain_rate}

Contributors The study was conceived and designed by AS, all the echo examinations, as well as some of the measurements were done by $\mathrm{HD}$ and the wall length and MAPSE measurements by HEM, while AS did the calculations and the geometric and statistical analyses. All of the authors contributed to the writing and revision of manuscript and approved the final draft.

Funding The study was fully sponsored by the Norwegian University of Science and Technology, as a PhD grant, as well as the HUNT Study providing the infrastructure for the Echocardiography in HUNT3 Study.

Competing interests None declared.

Patient consent for publication Not required.

Ethics approval The study was approved by the Regional Committee for Ethics in Medicine and Research (REK Mid-Norway 4.2009 .397 and 2018/929).

Provenance and peer review Not commissioned; externally peer reviewed.

Data availability statement Data are available on reasonable request. Deidentified patient data are deposited in the HUNT database. Echo data are in the department database (NTNU department of circulation and medical imaging).

Open access This is an open access article distributed in accordance with the Creative Commons Attribution Non Commercial (CC BY-NC 4.0) license, which permits others to distribute, remix, adapt, build upon this work non-commercially, and license their derivative works on different terms, provided the original work is properly cited, appropriate credit is given, any changes made indicated, and the use is non-commercial. See: http://creativecommons.org/licenses/by-nc/4.0/.

ORCID iD

Asbjørn Støylen http://orcid.org/0000-0002-2245-7066

\section{REFERENCES}

1 Støylen A, Mølmen HE, Dalen H. Left ventricular global strains by linear measurements in three dimensions: interrelations and relations to age, gender and body size in the HUNT study. Open Heart 2019;6:e001050.

2 Palmon LC, Reichek N, Yeon SB, et al. Intramural myocardial shortening in hypertensive left ventricular hypertrophy with normal pump function. Circulation 1994;89:122-31.

3 Lundbäck S. Cardiac pumping and function of the ventricular septum. Acta Physiol Scand Suppl 1986;550:1-101.

4 Emilsson K, Brudin L, Wandt B. The mode of left ventricular pumping: is there an outer contour change in addition to the atrioventricular plane displacement? Clin Physiol 2001;21:437-46.

5 Carlsson M, Cain P, Holmqvist C, et al. Total heart volume variation throughout the cardiac cycle in humans. Am J Physiol Heart Circ Physiol 2004;287:H243-50.

6 Ballo P, Quatrini I, Giacomin E, et al. Circumferential versus longitudinal systolic function in patients with hypertension: a nonlinear relation. J Am Soc Echocardiogr 2007;20:298-306.

7 Carlhäll C, Wigström L, Heiberg E, et al. Contribution of mitral annular excursion and shape dynamics to total left ventricular volume change. Am J Physiol Heart Circ Physiol 2004;287:H1836-41.

8 Carlsson $\mathrm{M}$, Ugander M, Mosén $\mathrm{H}$, et al. Atrioventricular plane displacement is the major contributor to left ventricular pumping in healthy adults, athletes, and patients with dilated cardiomyopathy. Am J Physiol Heart Circ Physiol 2007;292:H1452-9.

9 Maciver $\mathrm{DH}$. The relative impact of circumferential and longitudinal shortening on left ventricular ejection fraction and stroke volume. Exp Clin Cardiol 2012;17:5-11.

10 Stokke TM, Hasselberg NE, Smedsrud MK, et al. Geometry as a confounder when assessing ventricular systolic function: comparison between ejection fraction and strain. J Am Coll Cardiol 2017;70:942-54.

11 Yingchoncharoen T, Agarwal S, Popović ZB, et al. Normal ranges of left ventricular strain: a meta-analysis. J Am Soc Echocardiogr 2013;26:185-91.

12 Emilsson K, Wandt B. The relation between mitral annulus motion and ejection fraction changes with age and heart size. Clin Physiol 2000;20:38-43.

13 Kuznetsova T, Herbots L, Richart T, et al. Left ventricular strain and strain rate in a general population. Eur Heart J 2008;29:2014-23.
14 Dalen H, Thorstensen A, Aase SA, et al. Segmental and global longitudinal strain and strain rate based on echocardiography of 1266 healthy individuals: the HUNT study in Norway. Eur $J$ Echocardiogr 2010;11:176-83.

15 Sun JP, Lee AP-W, Wu C, et al. Quantification of left ventricular regional myocardial function using two-dimensional speckle tracking echocardiography in healthy volunteers--a multi-center study. Int $J$ Cardiol 2013;167:495-501.

16 Kou S, Caballero L, Dulgheru R, et al. Echocardiographic reference ranges for normal cardiac chamber size: results from the NORRE study. Eur Heart J Cardiovasc Imaging 2014;15:680-90.

17 Sugimoto T, Dulgheru R, Bernard A, et al. Echocardiographic reference ranges for normal left ventricular 2D strain: results from the EACVI NORRE study. Eur Heart J Cardiovasc Imaging 2017;18:833-40.

18 Støylen A, Mølmen HE, Dalen H. Relation between mitral annular plane systolic excursion and global longitudinal strain in normal subjects: the HUNT study. Echocardiography 2018;35:603-10.

19 Nikitin NP, Witte KKA, Ingle L, et al. Longitudinal myocardial dysfunction in healthy older subjects as a manifestation of cardiac ageing. Age Ageing 2005;34:343-9.

20 Mizuguchi $\mathrm{Y}$, Oishi $\mathrm{Y}$, Miyoshi $\mathrm{H}$, et al. The functional role of longitudinal, circumferential, and radial myocardial deformation for regulating the early impairment of left ventricular contraction and relaxation in patients with cardiovascular risk factors: a study with two-dimensional strain imaging. J Am Soc Echocardiogr 2008;21:1138-44.

21 Geyer H, Caracciolo G, Abe H, et al. Assessment of myocardial mechanics using speckle tracking echocardiography: fundamentals and clinical applications. J Am Soc Echocardiogr 2010;23:351-69.

22 Mor-Avi V, Lang RM, Badano LP, et al. Current and evolving echocardiographic techniques for the quantitative evaluation of cardiac mechanics: ASE/EAE consensus statement on methodology and indications endorsed by the Japanese Society of echocardiography. Eur J Echocardiogr 2011;12:167-205.

23 Gerstenblith G, Frederiksen J, Yin FC, et al. Echocardiographic assessment of a normal adult aging population. Circulation 1977:56:273-8.

24 Henry WL, Gardin JM, Ware JH. Echocardiographic measurements in normal subjects from infancy to old age. Circulation 1980;62:1054-61.

25 Devereux RB, Lutas EM, Casale PN, et al. Standardization of Mmode echocardiographic left ventricular anatomic measurements. $J$ Am Coll Cardiol 1984;4:1222-30.

26 Knutsen KM, Stugaard M, Michelsen S, et al. M-mode echocardiographic findings in apparently healthy, non-athletic Norwegians aged 20-70 years. Influence of age, sex and body surface area. J Intern Med 1989;225:111-5.

27 Ganau A, Saba PS, Roman MJ, et al. Ageing induces left ventricular concentric remodelling in normotensive subjects. $J$ Hypertens 1995:13:1818???1822-22.

28 Støylen A, Mølmen HE, Dalen H. Importance of length and external diameter in left ventricular geometry. normal values from the HUNT study. Open Heart 2016;3:e000465.

29 Aurigemma GP, Gaasch WH, McLaughlin M, et al. Reduced left ventricular systolic pump performance and depressed myocardial contractile function in patients $>65$ years of age with normal ejection fraction and a high relative wall thickness. Am J Cardiol 1995;76:702-5.

30 Serri K, Reant P, Lafitte M, et al. Global and regional myocardial function quantification by two-dimensional strain: application in hypertrophic cardiomyopathy. J Am Coll Cardiol 2006;47:1175-81.

31 Mizuguchi $\mathrm{Y}$, Oishi $\mathrm{Y}$, Miyoshi $\mathrm{H}$, et al. Concentric left ventricular hypertrophy brings deterioration of systolic longitudinal, circumferential, and radial myocardial deformation in hypertensive patients with preserved left ventricular pump function. J Cardiol 2010;55:23-33.

32 Kraigher-Krainer E, Shah AM, Gupta DK, et al. Impaired systolic function by strain imaging in heart failure with preserved ejection fraction. J Am Coll Cardiol 2014;63:447-56.

33 Lang RM, Badano LP, Mor-Avi V, et al. Recommendations for cardiac chamber quantification by echocardiography in adults: an update from the American Society of echocardiography and the European association of cardiovascular imaging. J Am Soc Echocardiogr 2015;28:1-39.

34 Shub C, Klein AL, Zachariah PK, et al. Determination of left ventricular mass by echocardiography in a normal population: effect of age and sex in addition to body size. Mayo Clin Proc 1994;69:205-11.

35 Hees PS, Fleg JL, Lakatta EG, et al. Left ventricular remodeling with age in normal men versus women: novel insights using 
three-dimensional magnetic resonance imaging. Am J Cardiol 2002;90:1231-6.

36 Zhu Y, Papademetris X, Sinusas AJ, et al. A coupled deformable model for tracking myocardial borders from real-time echocardiography using an incompressibility constraint. Med Image Anal 2010;14:429-48.

37 Dalen H, Thorstensen A, Romundstad PR, et al. Cardiovascular risk factors and systolic and diastolic cardiac function: a tissue Doppler and speckle tracking echocardiographic study. J Am Soc Echocardiogr 2011;24:322-32.

38 Thorstensen A, Dalen H, Amundsen BH, et al. Reproducibility in echocardiographic assessment of the left ventricular global and regional function, the HUNT study. Eur J Echocardiogr 2010;11:149-56.

39 Midthjell K, Lee CMY, Langhammer A, et al. Trends in overweight and obesity over 22 years in a large adult population: the HUNT study, Norway. Clin Obes 2013;3:12-20.

40 Simonson JS, Schiller NB. Descent of the base of the left ventricle: an echocardiographic index of left ventricular function. J Am Soc Echocardiogr 1989;2:25-35.

41 Höglund C, Alam M, Thorstrand C. Atrioventricular valve plane displacement in healthy persons. An echocardiographic study. Acta Med Scand 1988;224:557-62.

42 Wandt B. Long-axis contraction of the ventricles: a modern approach, but described already by Leonardo dA Vinci. J Am Soc Echocardiogr 2000;13:699-706.

43 Hamilton WF, Rompf JH. Movements of the base of the ventricle and the relative constancy of the cardiac volume. Am J Physiol 1932;102:559-65.
44 Hoffman EA, Ritman EL. Invariant total heart volume in the intact thorax. Am J Physiol 1985;249:H883-90.

45 Ugander M, Carlsson M, Arheden H. Short-axis epicardial volume change is a measure of cardiac left ventricular short-axis function, which is independent of myocardial wall thickness. Am J Physiol Heart Circ Physiol 2010;298:H530-5.

46 Carlsson M, Ugander M, Heiberg E, et al. The quantitative relationship between longitudinal and radial function in left, right, and total heart pumping in humans. Am J Physiol Heart Circ Physiol 2007;293:H636-44.

47 Emilsson K, Egerlid R, Nygren B-M, et al. Mitral annulus motion versus long-axis fractional shortening. Exp Clin Cardiol 2006;11:302-4.

48 Devereux RB, de Simone G, Ganau A, et al. Left ventricular hypertrophy and geometric remodeling in hypertension: stimuli, functional consequences and prognostic implications. J Hypertens Suppl 1994;12:S117-27

49 Stewart MH, Lavie CJ, Shah S, et al. Prognostic implications of left ventricular hypertrophy. Prog Cardiovasc Dis 2018;61:446-55.

50 Dalen $\mathrm{H}$, Thorstensen A, Vatten LJ, et al. Reference values and distribution of conventional echocardiographic Doppler measures and longitudinal tissue Doppler velocities in a population free from cardiovascular disease. Circ Cardiovasc Imaging 2010;3:614-22.

51 Höglund C, Alam M, Thorstrand C. Effects of acute myocardial infarction on the displacement of the atrioventricular plane: an echocardiographic study. J Intern Med 1989;226:251-6. 